Influence of metal organic chemical vapour deposition growth conditions on vibrational and luminescent properties of $\mathrm{ZnO}$ nanorods

D. N. Montenegro, V. Hortelano, O. Martínez, M. C. Martínez-Tomas, V. Sallet, V. Muñoz-Sanjosé, and J. Jiménez

Citation: Journal of Applied Physics 113, 143513 (2013); doi: 10.1063/1.4801534

View online: https://doi.org/10.1063/1.4801534

View Table of Contents: http://aip.scitation.org/toc/jap/113/14

Published by the American Institute of Physics

Articles you may be interested in

A comprehensive review of $\mathrm{ZnO}$ materials and devices

Journal of Applied Physics 98, 041301 (2005); 10.1063/1.1992666

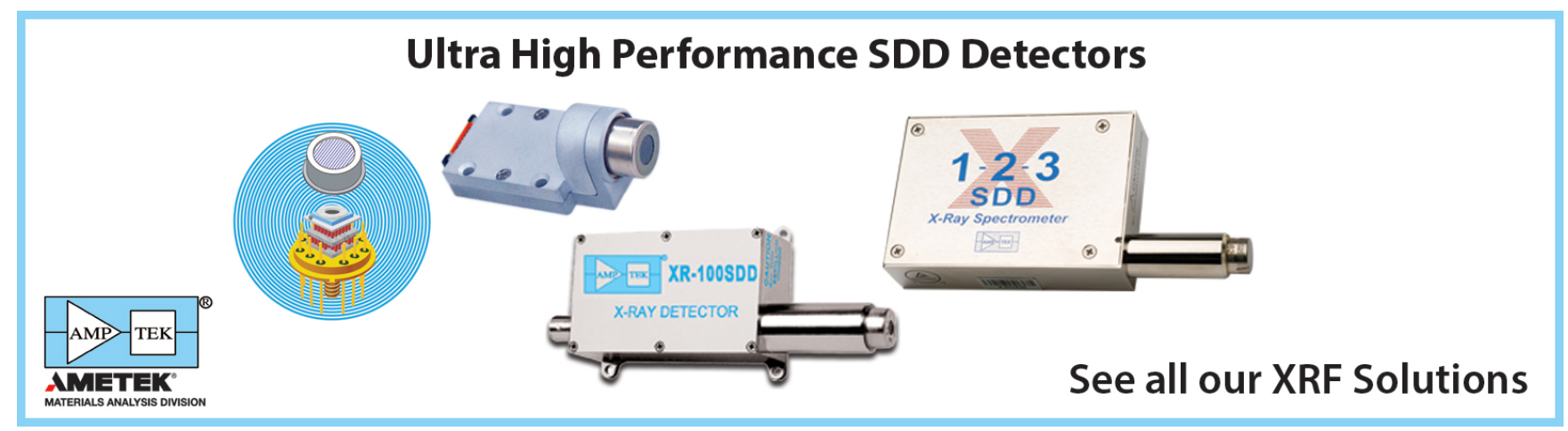




\title{
Influence of metal organic chemical vapour deposition growth conditions on vibrational and luminescent properties of $\mathrm{ZnO}$ nanorods
}

\author{
D. N. Montenegro, ${ }^{1, a)}$ V. Hortelano, ${ }^{2}$ O. Martínez, ${ }^{2}$ M. C. Martínez-Tomas, ${ }^{1}$ V. Sallet,${ }^{3}$ \\ V. Muñoz-Sanjosé, ${ }^{1}$ and J. Jiménez ${ }^{2}$ \\ ${ }^{1}$ Departamento de Física Aplicada y Electromagnetismo, Universidad de Valencia, Dr. Moliner 50, \\ 46100 Burjassot, Spain \\ ${ }^{2}$ GdS-Optronlab, Departamento de Física de la Materia Condensada, Edificio I $+D$, \\ Universidad de Valladolid, Paseo de Belén 1, 47011, Valladolid, Spain \\ ${ }^{3}$ Groupe d'Etude de la Matière Condensée (GEMAC), CNRS-Université de Versailles St-Quentin, \\ 45 avenue des Etats-Unis, 78035 Versailles Cedex, France
}

(Received 28 January 2013; accepted 27 March 2013; published online 12 April 2013)

\begin{abstract}
A detailed optical characterization by means of micro Raman and cathodoluminescence spectroscopy of catalyst-free $\mathrm{ZnO}$ nanorods grown by atmospheric-metal organic chemical vapour deposition has been carried out. This characterization has allowed correlating the growth conditions, in particular the precursors partial-pressures and growth time, with the optical properties of nanorods. It has been shown that a high $\mathrm{Zn}$ supersaturation can favor the incorporation of nonradiative recombination centers, which can tentatively be associated with $Z n_{I}$-related defects. Characterization of individual nanorods has evidenced that $Z n_{I}$-related defects have a tendency to accumulate in the tip part of the nanorods, which present dark cathodoluminescence contrast with respect to the nanorods bottom. The effect of a $\mathrm{ZnO}$ buffer layer on the properties of the nanorods has been also investigated, showing that the buffer layer improves the luminescence efficiency of the $\mathrm{ZnO}$ nanorods, revealing a significant reduction of the concentration of nonradiative recombination centers. (C) 2013 AIP Publishing LLC [http://dx.doi.org/10.1063/1.4801534]
\end{abstract}

\section{INTRODUCTION}

One-dimensional $\mathrm{ZnO}$ nanostructures have attracted intensive research interest due to its wide variety of technological applications. ${ }^{1,2}$ In particular, ZnO-based light emitting nanodiodes (LEDs) have been a matter of interest due to the large spectral range they could cover. ${ }^{3,4}$ The properties of $\mathrm{ZnO}$ such as the direct band-gap energy $(3.37 \mathrm{eV}$ at room temperature) and the large free exciton binding energy $(60 \mathrm{meV})$, in addition to the presence of deep centers coming from intrinsic and/or extrinsic defects, allow $\mathrm{ZnO}$ nanostructures to exhibit light emitting in violet, blue, green, yellow, and orange-red spectral ranges, covering almost the whole visible spectral window. ${ }^{5,6}$ Nevertheless, the intrinsic and/or extrinsic defects can also promote the formation of nonradiative recombination centers (NRRCs) which significantly reduce the internal quantum efficiency of luminescence emission. ${ }^{7}$ Many studies have evidenced the influence of the growth method or/and growth conditions on the optical properties of $\mathrm{ZnO}$ nanostructures. ${ }^{8,9}$ Although extensive information of $\mathrm{ZnO}$ defects can be found in the literature, ${ }^{10,11}$ the origin of the deep level luminescence emission (DLE) and NRRCs in $\mathrm{ZnO}$ still continues to be a matter of controversy and study. Therefore the control of the defect incorporation in $\mathrm{ZnO}$ nanorods or nanowires during the growth process is a main concern to be understood in order to improve the performance of $\mathrm{ZnO}$-based nanodevices.

\footnotetext{
a) Author to whom correspondence should be addressed. Electronic mail: Diana.Montenegro@uv.es. Tel.: +34 9635 44908. Fax: +34 963543146.
}

$\mathrm{ZnO}$ nanorod and nanowire arrays have been synthesized using several growth methods involving in most cases the transport of precursors in the vapour phase ${ }^{12-14}$ or wet chemistry processes. ${ }^{15-17}$ The simplicity of some of the used growth methods allows to reduce the costs, which constitutes a relevant industrial advantage. However, in some cases, the control of the growth conditions is considerably limited, and the synthesis of $\mathrm{ZnO}$ nanowire and nanorod arrays, with good crystalline quality and reproducibility, is difficult to achieve. Metal organic chemical vapour deposition (MOCVD) is a method allowing an accurate control of the growth parameters in addition to be highly adaptable to the synthesis of vertically well aligned $\mathrm{ZnO}$ nanorod arrays with good structural properties, controllable aspect ratio (length/width), and high reproducibility, ${ }^{18,19}$ without renouncing to its industrial vocation.

In this work, we present a comprehensive study of the influence of MOCVD-growth parameters on the optical properties of free-catalyst $\mathrm{ZnO}$ nanorod arrays, characterized by micro-Raman and cathodoluminescence (CL) spectroscopy. We have analyzed the effect of the precursor's partialpressure ratio $\left(R_{V I / I I}\right)$, growth time, and the use of a $\mathrm{ZnO}$ buffer layer on the synthesis process. Global characterization of $\mathrm{ZnO}$ nanorod arrays by means of both types of spectroscopic tools has allowed to correlate the observed red-shift of the DLE luminescence in the CL spectrum and the existence of NRRCs associated with the presence of $Z n_{I}$-related defects.

Likewise, the characterization of individual nanorods has allowed to focus on the location and distribution of these defects along the nanorods. The effect of the deposition of a 
buffer layer, previous to the growth of the nanorods, on their optical properties has revealed that this layer seems to reduce significantly the incorporation of $Z n_{I}$-related defects, improving the internal quantum efficiency.

\section{EXPERIMENTAL SECTION}

Vertically well aligned $\mathrm{ZnO}$ nanorods were grown on $c$-sapphire substrates with and without a $\mathrm{ZnO}$ buffer layer in an atmospheric MOCVD reactor. The buffer layer was grown using hydrogen as carrier gas and DMZn-TEN and t$\mathrm{BuOH}$ as zinc and oxygen precursors, respectively. $\mathrm{ZnO}$ nanorods arrays were synthesized using helium as carrier gas and $\mathrm{N}_{2} \mathrm{O}$ as oxygen precursor. The $\mathrm{ZnO}$ buffer layer was grown at $450{ }^{\circ} \mathrm{C}$ during $30 \mathrm{~min}$. A wide variation of the $R_{V I / I I}$ (80 to 300 ) was made by changing individually the precursors' flow-rates. In sample series \#1, the $\mathrm{N}_{2} \mathrm{O}$ flow-rate was changed from 300 to $900 \mathrm{sccm}$, being the DMZn-TEN flowrate kept constant at $75 \mathrm{sccm}$. For the sample series \#2, the DMZn-TEN flow-rate was varied from 15 to $95 \mathrm{sccm}$, while the $\mathrm{N}_{2} \mathrm{O}$ flow-rate was set at $300 \mathrm{sccm}$. On the other hand, the sample series \#3 corresponds to the simultaneous variation of both precursors' flow-rates while keeping constant the $R_{V I / I I}$ at 100 . Morphological characteristics and details of growth mechanisms of this type of samples can be found in our previous work. ${ }^{20}$ The growth time for nanorods grown on a buffer layer on $c$-sapphire was varied from 10 to $40 \mathrm{~min}$, while for nanorods grown on bare substrates it was varied from 5 to $15 \mathrm{~min}$. All the $\mathrm{ZnO}$ nanorods were synthesized at $800^{\circ} \mathrm{C}$.

The Raman spectra were acquired at room temperature using the $532 \mathrm{~nm}$ line of a frequency doubled Nd-YAG laser. The scattered light was analyzed by means of a Raman spectrometer (Labram HR800 UV from Horiba-Jobin-Yvon) equipped with a $\mathrm{LN}_{2}$-cooled charge-coupled device (CCD) detector. CL measurements were carried out at $80 \mathrm{~K}$ with a Gatan MonoCL2 system attached to a field emission scanning electron microscope (FESEM) (LEO 1530). The acceleration voltage of the e-beam was varied between 3 and $20 \mathrm{kV}$. The CL spectra were acquired using a Peltier cooled
$\mathrm{CCD}$ as a detector. The morphology of $\mathrm{ZnO}$ nanorods was assessed using scanning electron microscopy (SEM) (Hitachi 4800).

\section{RESULTS AND DISCUSSION}

This section has been divided into two subsections, in which we analyze the $\mathrm{ZnO}$ nanorods grown on $c$-sapphire substrates, without (Subsection III A) and with (Subsection III B) a $\mathrm{ZnO}$ buffer layer. In the first subsection, the effect of the precursor partial-pressure ratio and the growth time on the optical properties of the nanorods is studied, while the second subsection concerns the effect of the $\mathrm{ZnO}$ buffer layer.

\section{A. $R_{V I / I}$ and growth time}

\section{Raman characterization}

Wurzite-type $\mathrm{ZnO}$ belongs to the space group $C^{4}{ }_{6 v}$ with two formula units in the primitive cell. The optical phonons at the $\Gamma$ point of the Brillouin zone correspond to the irreducible representation: $\Gamma_{\text {opt }}=A_{1}+E_{1}+2 E_{2}+2 B_{1} .^{21}$ The polar modes $A_{1}$ and $E_{1}$ are split out into transverse optical (TO) and longitudinal optical (LO) phonons, both modes are Raman and infrared active. $E_{2}$ modes are non-polar and are only Raman active. $B_{1}$ modes are Raman and infrared silent modes.

Figure 1(a) shows a typical Raman spectrum of $\mathrm{ZnO}$ nanorods arrays grown on $c$-sapphire substrates under a precursor partial-pressures ratio $R_{V I / I I} \sim 100$ (corresponding to a $\mathrm{N}_{2} \mathrm{O}$ flow-rate of $300 \mathrm{sccm}$ and a DMZn-TEN flow-rate of $75 \mathrm{sccm})$. A set of well-defined peaks can be observed in this spectrum. The peaks at 333 and $437 \mathrm{~cm}^{-1}$ are commonly assigned to second order and $E_{2}{ }^{(h i g h)}$ modes, respectively. $^{22,23}$ The additional peaks at 275,510 , and $643 \mathrm{~cm}^{-1}$ are not attributed to $\mathrm{ZnO}$ normal modes. These peaks have been related to intrinsic defects or impurities in the material, although their exact origin still remains under debate. The peak around $580 \mathrm{~cm}^{-1}$ has been ascribed to the $E_{1}(\mathrm{LO})$ mode of $\mathrm{ZnO}$, allowed for the $x(z y) \bar{x}$ and $x(z y) y$ scattering geometries. ${ }^{23}$ However, some authors have observed this peak in

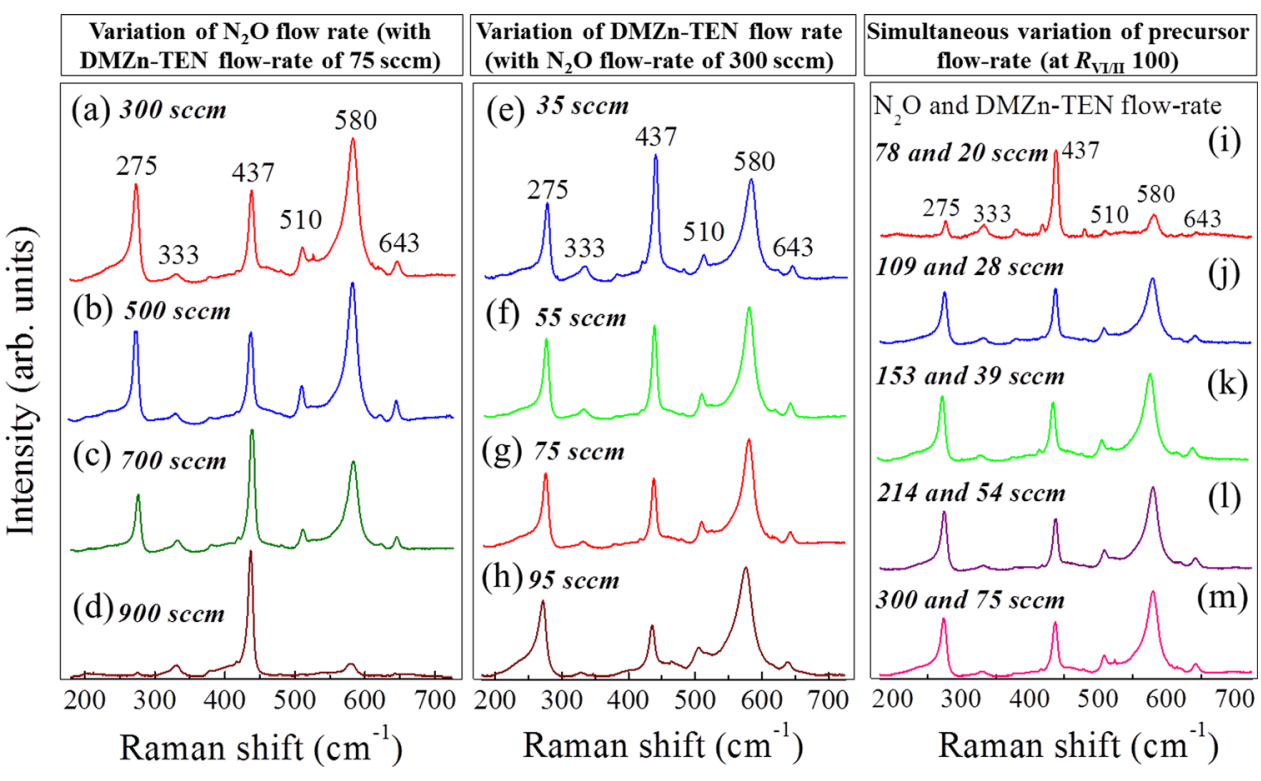

FIG. 1. Raman spectra of $\mathrm{ZnO}$ nanorod arrays grown with: (a)-(d) different $R_{V I / I I}$ due to the variation of $\mathrm{N}_{2} \mathrm{O}$ flow-rate (series \#1); (e)-(h) different $R_{V I / I I}$ due to the variation of DMZn-TEN flow-rate (series \#2) and (i)-(m) the same $R_{V I / I I}$ $\approx 100$ but different precursors' flow-rates (series \#3). 
backscattering geometry $(z(x x) \bar{z})$ due to resonantly enhanced LO phonons. ${ }^{24}$ It should be noted that several scattering geometries coexist when measuring the Raman spectrum of nanostructure arrays.

In respect to the defect related bands, from the beginning of the last decade several studies have associated the Raman peaks at $275,510,580$, and $643 \mathrm{~cm}^{-1}$ with the incorporation of nitrogen impurities in $\mathrm{ZnO}$ thin films and nanostructures. ${ }^{25-27}$ Nevertheless, Bundesman et al. ${ }^{28}$ have reported that these peaks can be also present in $\mathrm{Fe}-, \mathrm{Sb}-, \mathrm{Al}-$, and Ga-doped $\mathrm{ZnO}$ samples, without traces of N. Otherwise, the occurrence of these defect-related peaks has been also reported in undoped $\mathrm{ZnO}$ samples, being attributed to $\mathrm{ZnO}$ silent modes. ${ }^{29}$ Recently, Friedrich et al. ${ }^{30}$ observed them in ion-implanted samples; combined with ab initio calculations, they associated the local vibrational modes located around 275 and $510 \mathrm{~cm}^{-1}$ with the formation of defect complexes constituted by impurity-related defects and zinc interstitials, e.g., of the type $\left(Z n_{I}-N_{O}\right)$ complexes for the peak at $275 \mathrm{~cm}^{-1}$, and of the type $\left(Z n_{I^{-}} O_{I}\right)$ complex for the peak at $510 \mathrm{~cm}^{-1}$.

In order to know more about the presence of the defect related Raman peaks at $275,510,580$, and $643 \mathrm{~cm}^{-1}$ in our nanorods, we have carried out a detailed study of the influence of the precursors partial-pressures ratio $\left(R_{V I / I I}\right)$ and growth time on the observation of these modes. Figures 1(a)-1(m) show the Raman spectra obtained for the three series of samples. The sample series \#1 and \#2 are obtained with different $R_{V I / I I}$ 's, which is done by changing independently the flow rate of oxygen or zinc precursors, as said before.

Significant differences can be observed in the relative intensities of the $E_{2}{ }^{(h i g h)}$ mode $\left(437 \mathrm{~cm}^{-1}\right)$ and the defectsrelated peaks $\left(275,510,580\right.$, and $\left.643 \mathrm{~cm}^{-1}\right)$ on the Raman spectra for the different precursors flow-rates. In order to highlight these differences in the defect-related peaks of Figure 1, relative intensities with respect to the $E_{2}{ }^{(h i g h)}$ mode have been considered. Figures 2(a) and 2(b) exhibit the change of these intensities as a function of the flow rate of oxygen and zinc precursors, respectively. In sample series \#1, the relative intensities of the defects-related peaks with respect to the $E_{2}{ }^{(h i g h)}$ mode are reduced and tend to disappear at high $\mathrm{N}_{2} \mathrm{O}$ flow-rates $(\sim 900 \mathrm{sccm})$. On the contrary, in the sample series \#2, regarding the variation of DMZn-TEN flow-rate, the relative intensities of the defect-related peaks with respect to the $E_{2}{ }^{(h i g h)}$ mode tend to increase with the flow rate. Figure 2(c) shows the variation on the relative intensities of each defectrelated peak as a function of the $R_{V I / I I}$. This behavior corroborates that the intensity of the defects-related peaks is enhanced when the growth is made under high zinc-rich conditions (low $R_{V I / I I}$ ), while it is considerably reduced under high oxygenrich conditions (high $R_{V I / I I}$ ).

Consequently, from these considerations and according to the behavior observed in Figures 1 and 2, it is possible to conclude that the local vibrational modes at 275, 510, and $643 \mathrm{~cm}^{-1}$ found in our samples seem to be associated with an excess of $\mathrm{Zn}$, which appears compatible with the formation of $\mathrm{Zn} n_{I}$-defects complexes into $\mathrm{ZnO}$ nanorods as claimed by other authors. ${ }^{30}$ Likewise, the enhancement exhibited by the $E_{1}$ (LO) mode at $580 \mathrm{~cm}^{-1}$ can be also ascribed to the formation of these $Z n_{I}$-related defects as this type of complex would produce the breaking of the $\mathrm{Zn}-\mathrm{O}$ bonds, giving rise to such $E_{1}(\mathrm{LO})$ mode. $^{31}$

Furthermore, the relative intensities of the defect-related peaks with respect to the $E_{2}{ }^{(h i g h)}$ mode in the spectra corresponding to sample series \#3 (i.e., samples grown at $R_{V I / I I}$ constant) have been analyzed. Figure 2(d) shows these relative intensities as a function of DMZn-TEN flow-rate. As it
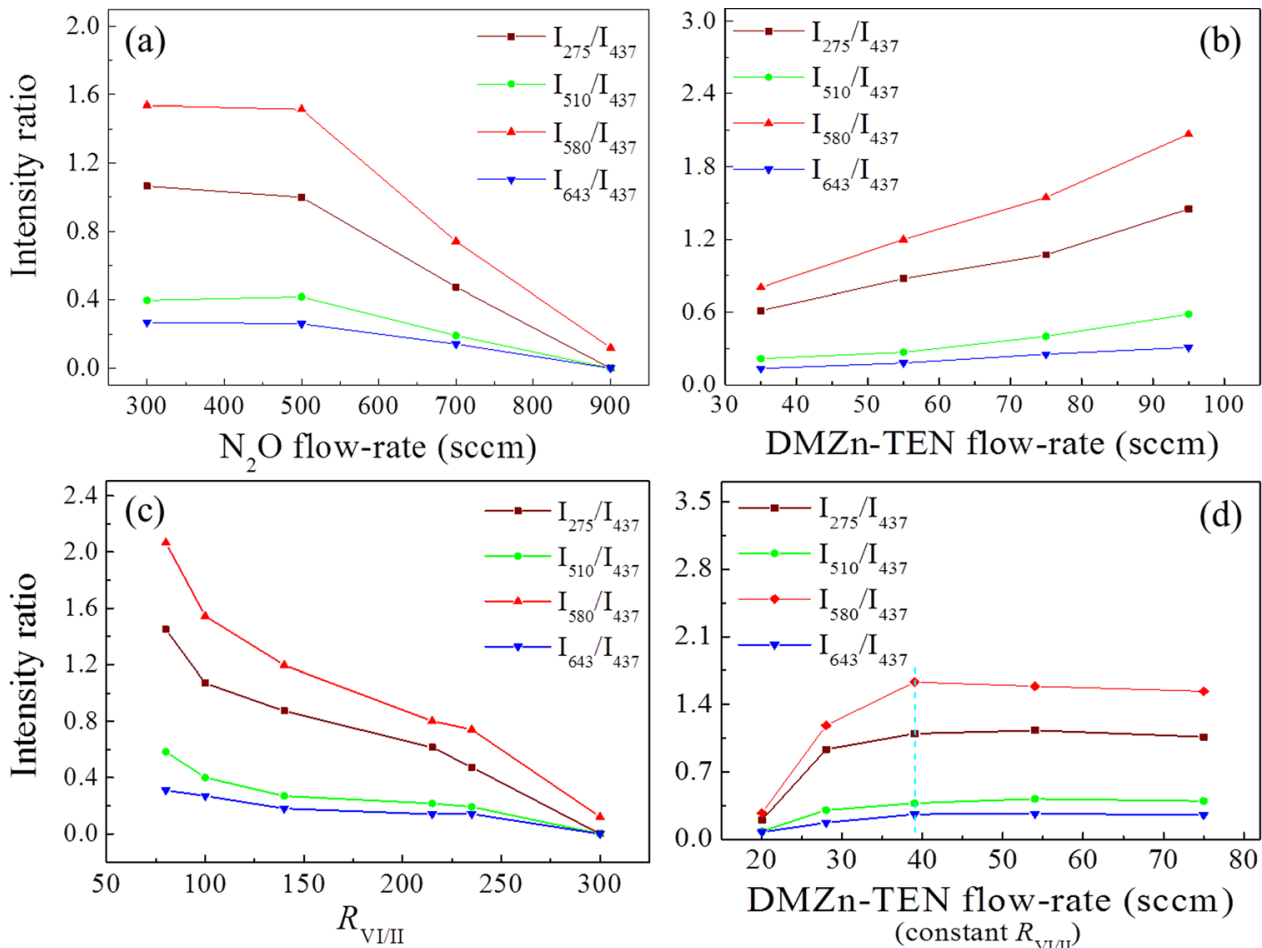

DMZn-TEN flow-rate (sccm)

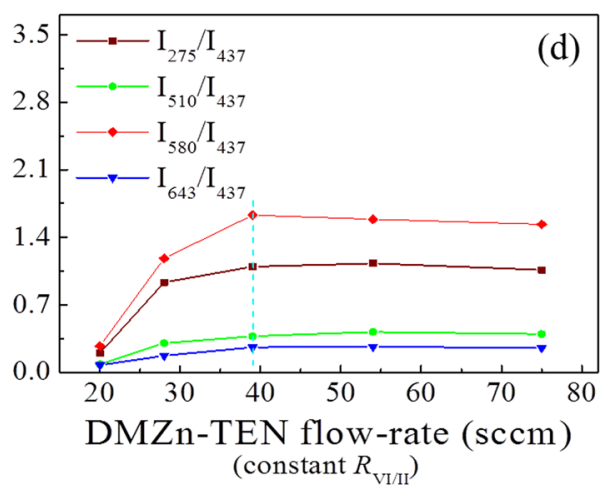

FIG. 2. Ratio of intensities of the defectsrelated Raman peaks with respect to the $E_{2}{ }^{\text {high }}$ Raman peak intensity as a function of (a) $\mathrm{N}_{2} \mathrm{O}$ flow-rate; (b) DMZn-TEN flow-rate; (c) precursors flow rate $R_{V I / I}$; and (d) DMZn-TEN flow-rate under constant $R_{V I / I I}$. 

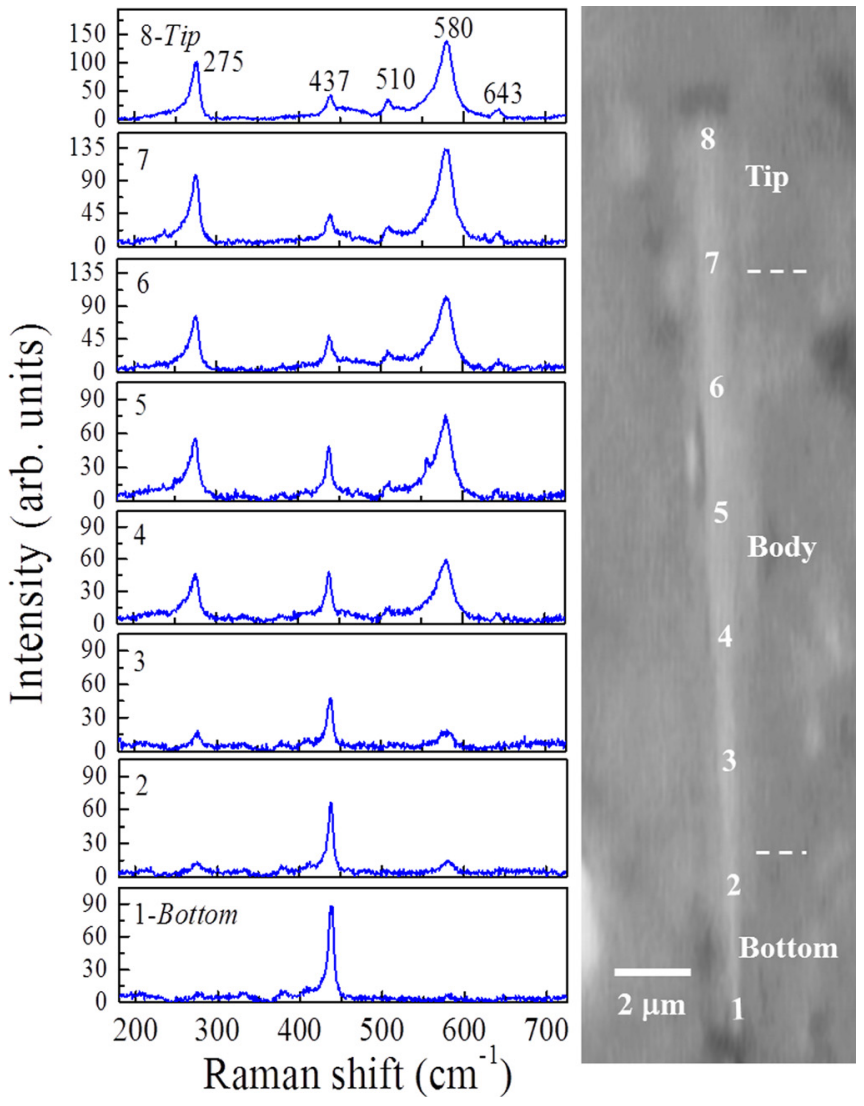

FIG. 3. (a) Micro-Raman spectra at different spatial positions along the length of a nanorod grown on bare $c$-sapphire.

can be seen, the relative intensities increase with the flow rate of zinc precursor until they reach a threshold from which they saturate. It is known that the variation of the precursor flow-rate has a direct influence on the growth rate of a material. Hence, from the observed behavior, we can extract two fundamental conclusions: (1) low precursor flow-rates (which give rise to low growth rates) tend to prevent or reduce the incorporation of $Z n_{I}$-related defects; (2) there is a threshold precursor flow-rate (which can be associated with a threshold growth rate), from which the incorporation of $Z n_{I}$-related defects seems to saturate.
The previous study has been carried out exciting arrays of nanorods. However, a complementary study focused on individual nanorods offers additional and relevant information on these nanostructures. Indeed, Figure 3 shows the Raman spectra acquired at different positions along the length of a single $\mathrm{ZnO}$ nanorod grown on a bare $c$-sapphire substrate. As it can be seen, the Raman spectrum changes along the nanorod. The intensities of defect-related peaks are gradually enhanced from bottom to top of the nanorod, suggesting that $Z n_{I}$-defect complexes are present in higher concentration at the nanorod tip. This result will be analyzed in correlation with the CL characterization.

\section{CL characterization}

A CL characterization has been made in order to correlate the growth conditions of nanorods to their luminescent properties. Figures 4(a) and 4(b) show CL spectra of samples of series \#1 and \#2. Typically, the luminescence spectrum of $\mathrm{ZnO}$ can be separated in three spectral sections: $\mathrm{E}>3.33 \mathrm{eV}$ corresponding to the near band edge (NBE) emission governed by the excitonic transitions, $3.33 \mathrm{eV}>\mathrm{E}>3.00 \mathrm{eV}$, which corresponds to free to bound, donor acceptor pair (DAP) transitions, and phonon replicas, and $\mathrm{E}<3.0 \mathrm{eV}$, which includes the DLE. The relevance of the different spectral contributions depends on the presence of impurities and defects.

For all the CL spectra recorded, two main peaks around 3.34 and $3.31 \mathrm{eV}$ are observed. The other peaks, with lower intensity, correspond to phonon replicas of the $3.31 \mathrm{eV}$ peak. In wurtzite-type $\mathrm{ZnO}$, the peaks located between 3.32 and $3.36 \mathrm{eV}$ are attributed to bound exciton transitions, being the most prominent lines labeled as I4, I6, and I9. ${ }^{32}$ The spectral resolution of our CL equipment could not resolve adequately these bound exciton transitions because the spectra were acquired at $80 \mathrm{~K}$, being thus observed a relatively broad band around $3.34 \mathrm{eV}$, which includes the different bound excitonic transitions and the free exciton emission in the high energy flank. The origin of the peak around $3.31 \mathrm{eV}$ remains controversial. It has been associated with the first LO phonon replica of the free exciton (1LO-FX); ${ }^{33,34}$ with a DAP

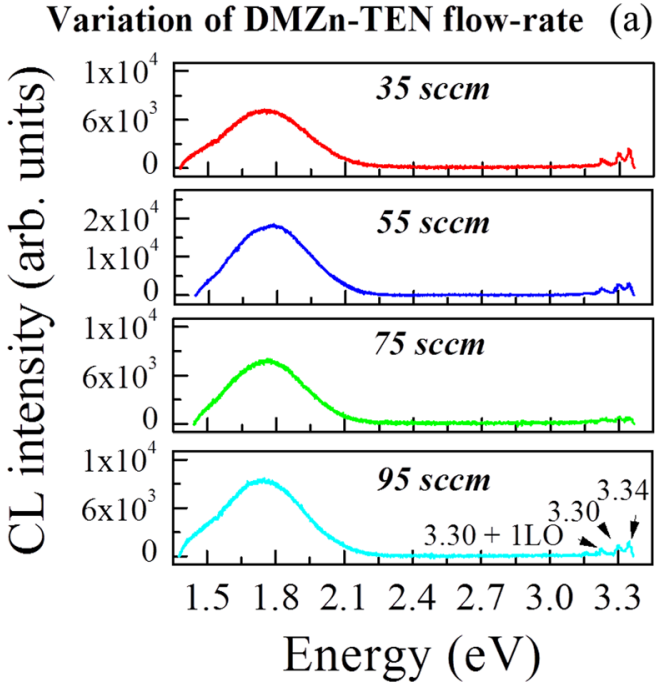

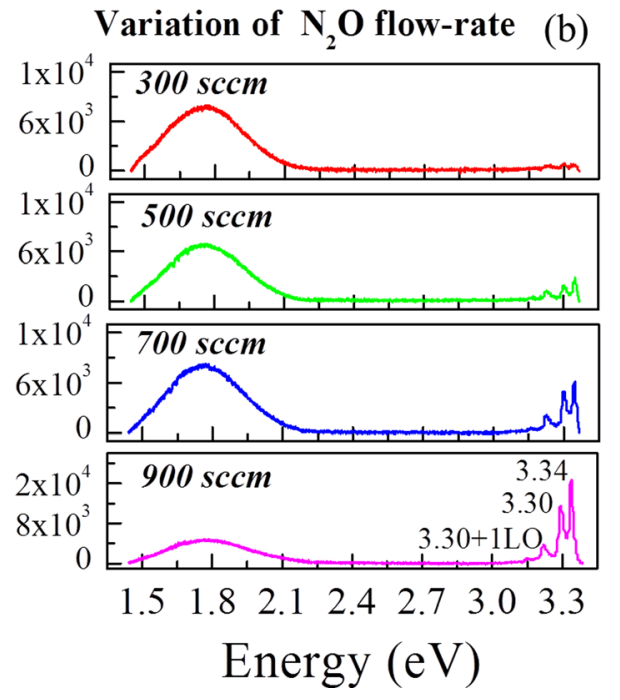

FIG. 4. CL spectra of $\mathrm{ZnO}$ nanorod arrays grown with different $R_{V I / I I}$ (a) due to the variation of DMZn-TEN flow-rate (series \#2); (b) due to the variation of $\mathrm{N}_{2} \mathrm{O}$ flow-rate (series \#1). 
transition; ${ }^{35}$ with a free to neutral acceptor $\left(\mathrm{e}-A^{0}\right)$ band $^{36}$ and with an exciton bound to extended defects (dislocations and staking faults). ${ }^{37}$

The DLE refers to a broad band extending from $2.8 \mathrm{eV}$ to $1.5 \mathrm{eV}$. The nanorods present all of them an unstructured broad band peaking at around $1.75 \mathrm{eV}$. The luminescence emission in the same spectral range has been previously reported in commercial $\mathrm{ZnO}$ bulk crystals, although the origin of this band is not fully understood. ${ }^{38}$ This red luminescence is not usually the dominant DLE in $\mathrm{ZnO}$, which normally presents a broad emission in the green-orange spectral range $(\sim 2-2.5 \mathrm{eV})$. Therefore, the DLE emission centered at $1.75 \mathrm{eV}$ is really characteristic of our nanorods. Although an extended debate remains about the origin of DLE luminescence in $\mathrm{ZnO}$, usually it is admitted that the green luminescence is associated with oxygen deficiency, namely, oxygen vacancies $\left(V_{O}\right),{ }^{39}$ the yellow-orange emission is usually associated with $\mathrm{Zn}$ deficiency, namely, zinc vacancies $\left(V_{Z n}\right)$ complexes or oxygen interstitials $\left(O_{I}\right){ }^{40}$ $\mathrm{ZnO}$ light emission in the red spectral range has been related to $\mathrm{Fe}$ - and N-impurities. ${ }^{41,42}$ In N-doped $\mathrm{ZnO}$, the $1.9 \mathrm{eV}$ band has been associated with a shallow donor to deep $\mathrm{N}$ acceptors transition; ${ }^{43}$ it has been also mentioned that $Z n_{I}$ is involved in the red emission, ${ }^{44}$ it could be the shallow donor of the DAP transition. Recent works have related the red luminescence to $V_{Z n}$ defects. ${ }^{10,45}$ In our nanorod samples, the presence of $Z n_{I}$ is largely consistent with the Raman results previously discussed, in which defect related local vibration modes were tentatively associated with the presence of $Z n_{I^{-}}$ related defects in the $\mathrm{ZnO}$ nanorods.

A relevant aspect to remark is the significant reduction of the NBE emission in the nanorods grown under high zinc precursor flow-rate. Following the evolution of the CL spectra with the variation of DMZn-TEN (Figure 4(a)), it can be observed that the intensity of the NBE emission is reduced for increasing zinc precursor flow-rate. Meanwhile, the intensity of the NBE emission increases with increasing the oxygen precursor flow-rate (Figure 4(b)). Nevertheless, increasing further the VI/II ratio leads to an evolution of the nanowires morphology towards $2 \mathrm{D}$ films. ${ }^{20}$

Moreover, the panchromatic CL images of the nanorods show that the nanorod bottom luminescence is bright and is progressively quenched when approaching the nanorod tip, Figure 5(a). This image reveals a strong change of the internal quantum efficiency along the nanorod. Monochromatic images centered at 3.34 and $1.75 \mathrm{eV}$ of an individual nanorod and acquired under different acceleration voltages (5 and $15 \mathrm{kV}$ ) are shown in Figure 5(b), both bands indistinctly decrease its intensity along the nanorod, indicating an increase of the NRRCs from bottom to tip.

NRRCs in $\mathrm{ZnO}$ have been related with the presence of defects such as $V_{Z n}$, which have been commonly invoked to produce NRRCs in the form of $V_{Z n}$-defect complexes, and/or surface defects or structural damage on the material. ${ }^{7}$ In our $\mathrm{ZnO}$ nanorods, the $\mathrm{CL}$ efficiency decreased with increasing DMZn-TEN flow-rate, and increased with increasing the $\mathrm{N}_{2} \mathrm{O}$ flow-rate, which suggests that the NRRCs are related to excess $\mathrm{Zn}$. On the other hand, from the micro-Raman characterization of individual $\mathrm{ZnO}$ nanorods, it has been concluded
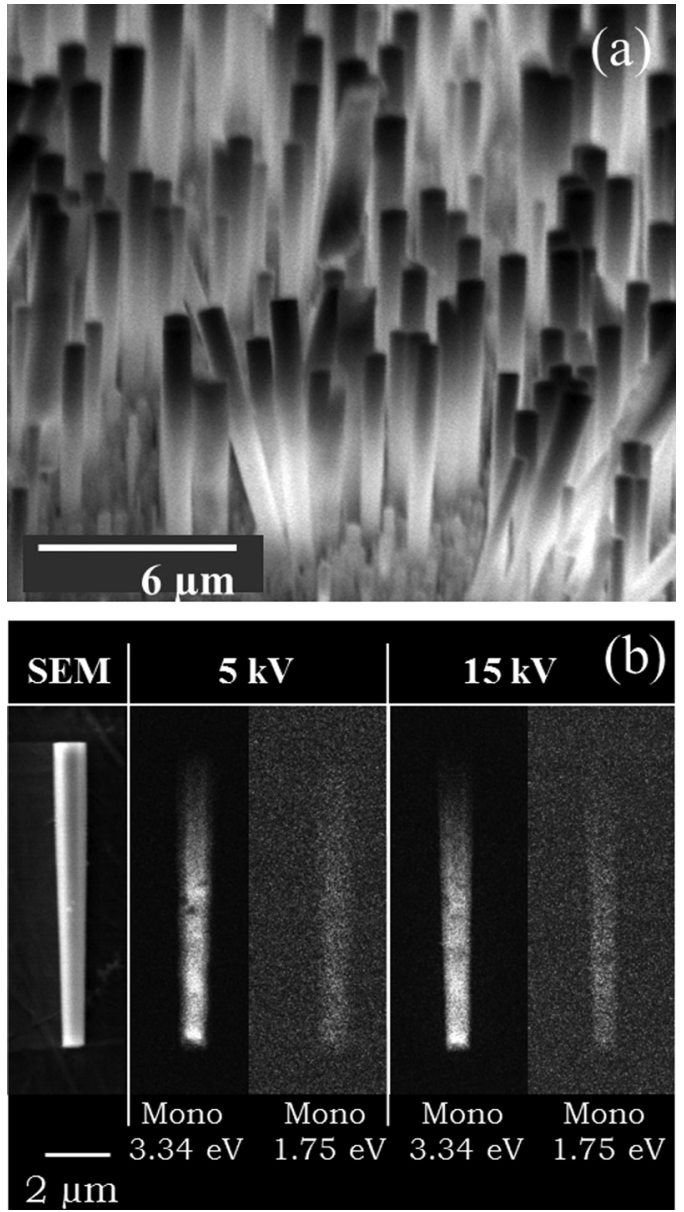

FIG. 5. (a) Panchromatic CL cross images of the $\mathrm{ZnO}$ nanorods arrays grown on bare $c$-sapphire; (b) SEM image and monochromatic CL images at 3.34 and $1.75 \mathrm{eV}$ of a single $\mathrm{ZnO}$ nanorod grown on bare $c$-sapphire acquired under 5 and $15 \mathrm{kV}$.

that $Z n_{I}$-defect complexes are mainly located at the nanorod's tip. Thus, it can be inferred that the strong reduction of the luminescence at nanorods tips could be related to the $Z n_{I}$-defect complexes, which will be the NRRCs. The other possibility is the formation of structural defects at the top part; however, HRTEM measurements did not reveal the presence of structural defects. ${ }^{46}$

Figure 6 shows the evolution of the CL spectrum along the length of two nanorods grown under different DMZnTEN flow-rates, 35 and $95 \mathrm{sccm}$, keeping constant the $\mathrm{N}_{2} \mathrm{O}$ flow-rate $(300 \mathrm{sccm})$, Figures 6(a) and 6(b), respectively. A similar evolution of the luminescence along both nanorods is observed: the intensity of the NBE emission is strongly damped at nanorods tips while the relative intensities of CL peaks are not significantly changed. However, the FWHM of the peaks and the signal/noise ratio are larger in the spectra belonging to the bigger nanorod (Figure 6(b)), which was grown under a higher zinc precursor flow-rate. The emission profiles along the length of both nanorods demonstrate that the emission efficiency is strongly reduced when the growth of these structures is made under high zinc supersaturation conditions, Figure 6(c).

In a previous work, we have demonstrated that high zinc supersaturation conditions favor the lateral growth of $\mathrm{ZnO}$ 

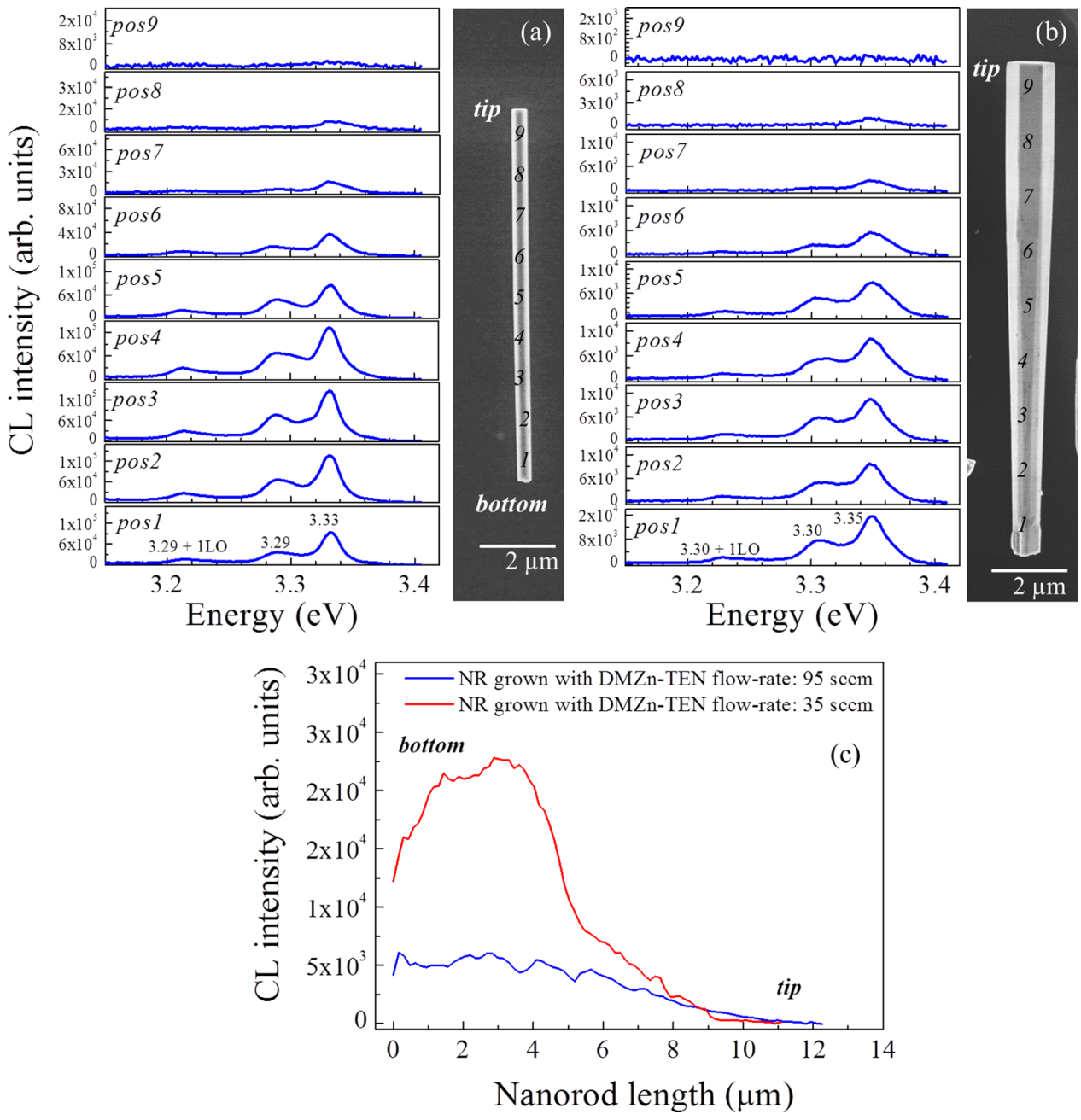

FIG. 6. CL spectra at different spatial positions along a nanorod grown on bare $c$-sapphire under $\mathrm{N}_{2} \mathrm{O}$ flow-rate of 300 sccm and DMZn-TEN flow-rates of (a) $35 \mathrm{sccm}$ and (b) $95 \mathrm{sccm}$. (c) CL intensity profile along the length of these nanorods. nanorods reducing their aspect ratio (length/width). ${ }^{20}$ In addition, on nanorods grown on bare sapphire we have observed that the lateral growth-rate at the nanorod tip is faster than at the nanorod bottom, giving rise to inverted conical nanorods for high $\mathrm{Zn}$ precursor flow-rates (Figure 6(b)), rather than the typical columnar nanorods (Figure 6(a)). In this frame, the accumulation of $Z n_{I}$-related defects at nanorods tips seems to be favored in this observed morphology of inverted cones. In order to go deeper into the correlation between the morphological evolution of nanorods and the incorporation of $Z n_{I}$-defects complex, a CL characterization of $\mathrm{ZnO}$ nanorods arrays synthesized for different growth times was done.

Figure 7 shows the cross sectional SEM (left) and panchromatic images (right) of $\mathrm{ZnO}$ nanorods grown under $R_{V I / I I} \sim 100\left(\mathrm{~N}_{2} \mathrm{O}\right.$ flow-rate: $300 \mathrm{sccm}$ and DMZn-TEN flowrate: $75 \mathrm{sccm}$ ) during 2, 5, and $15 \mathrm{~min}$ (up to down). The panchromatic images show remarkable changes on the luminescence of the $\mathrm{ZnO}$ nanorods depending on the growth time. For low growth time ( 2 min, Figure $7(d)$ ), a homogeneous luminescence is observed along the synthesized $\mathrm{ZnO}$ nanorods. When the growth time is increased to $5 \mathrm{~min}$ (Figure 7(e)), some dark nanorods tips can be distinguished, making evident the incorporation of NRRCs. Finally, under the larger growth time (15 min, Figure $7(f))$, the whole ensemble of grown nanorods exhibits dark tips. Figure 8(a) shows the CL intensity of the peak around $3.34 \mathrm{eV}$ as a function of the growth time. This graph reveals that the incorporation of NRRCs increases along the growth run; because the NRRCs seem to be related to excess $\mathrm{Zn}$, it could be argued that there is an effective increase of the $\mathrm{Zn}$ flow rate along the growth run. This is coherent with the observed CL intensity profile along the nanorod for two different DMZn-TEN flow-rates shown in Figure 6(c); we can observe a CL intensity decrease from bottom to tip, consequence of the enhanced incorporation of NRRCs, as well as lower CL intensity along the nanorod for the higher DMZn-TEN flowrate. Only in the quenched region close to the tip the intensities are equivalent for the two nanorods. Note that the change in the effective flow-rate could be a consequence of the morphologic evolution of the $\mathrm{ZnO}$ nanorods during the growth process. Simultaneously, a decrease of the $3.31 \mathrm{eV}$ band can be detected, see the spectra along the nanorods in Figures 6(a) and 6(b), which accounts for the overall defect presence, in agreement with the decrease of the $E_{2}{ }^{(h i g h)}$ Raman band, Figure 3.

The insets in the SEM images, Figures 7(a)-7(c), show the morphology evolution of nanorods with the growth time. It can be observed that the evolution of the nanorods diameter is not uniform, i.e., the lateral growth at the nanorod tip is faster than at the bottom. This behavior demonstrates that the morphology evolution of the nanorods with respect to the 

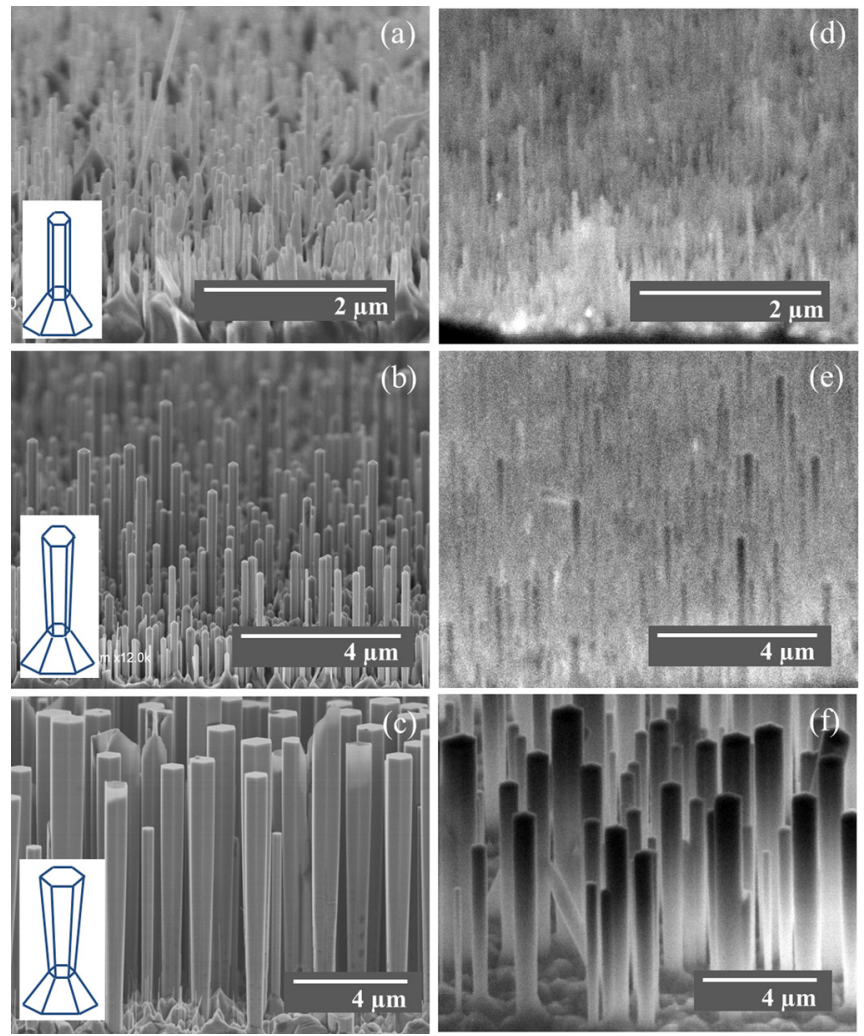

FIG. 7. SEM (left) and panchromatic CL (right) cross view images of $\mathrm{ZnO}$ nanorod arrays grown with a $R_{V I / I I} \approx 100$ at different growth times, 2 (a), (d); 5 (b), (e); and 15 (c), (f) minutes.

growth time is similar to the evolution shown with respect to the zinc precursor flow-rate. The evolution of the aspect ratio (length/width) of $\mathrm{ZnO}$ nanorods as a function of growth time is also plotted in Figure 8(a). In this case, only the diameter of nanorods tips has been taken into account as a reference for the variation of the nanorods width. The decreasing aspect ratio as the growth time increases shows that during the early growth stages $\left(t_{\mathrm{g}} \leq 2 \mathrm{~min}\right)$ the growth rate in the $c$-plane direction is dominant against the lateral growth. During this time (2 min), nanorods reach lengths of about $1-2 \mu \mathrm{m}$ and only small diameters between 80 and $150 \mathrm{~nm}$. Afterwards, when the growth time is increased, the lateral growth rate of the nanorod tip acquires more relevance and after $15 \mathrm{~min}$ the nanorods reach a diameter at the tip as large as $1-1.5 \mu \mathrm{m}$, while the length has only increased to $9 \mu \mathrm{m}$.

Lateral growth is enhanced for long growth times and seems to be responsible for the formation and accumulation of $Z n_{I}$-related defects at nanorods tips. In an attempt to explain this, the anisotropic growth that gives rise to the formation of nanorods must be considered. In $\mathrm{ZnO}$, longitudinal growth involves C-plane, and lateral growth, M- (or A-) plane. Assuming that growth processes, i.e., adsorption of molecules, mobility and incorporation of atoms, depend on the surface orientation, a growing M-plane could incorporate more $Z n_{I}$ defects than a growing C-plane surface, as it can be the case when doping a semiconductor with an impurity. Then, a higher level of NRRC can be found at the tip of the nanorod where lateral growth was predominant.

\section{B. ZnO buffer layer effect}

It is commonly admitted that the use of a $\mathrm{ZnO}$ buffer layer in the growth process of $\mathrm{ZnO}$ nanorods arrays improves their morphological and structural properties. ${ }^{47,48}$ In this subsection, we analyze the luminescence properties of $\mathrm{ZnO}$ nanorods arrays grown using a $\mathrm{ZnO}$ buffer layer.

Figure 8(b) shows the Raman spectra of $\mathrm{ZnO}$ nanorods arrays grown with and without a $\mathrm{ZnO}$ buffer layer during 20 min under $\mathrm{N}_{2} \mathrm{O}$ flow-rate of $800 \mathrm{sccm}$ and DMZn-TEN of 30 sccm. The spectrum from nanorod arrays grown on the $\mathrm{ZnO}$ buffer layer (buffered sapphire) does not exhibit the defectrelated peaks at $275,510,580$, and $643 \mathrm{~cm}^{-1}$, while they
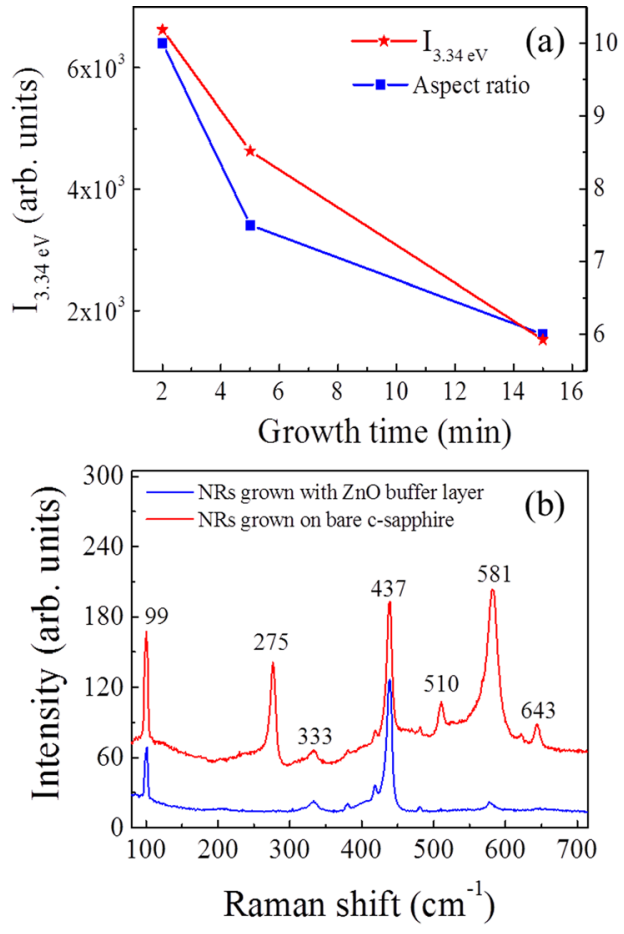

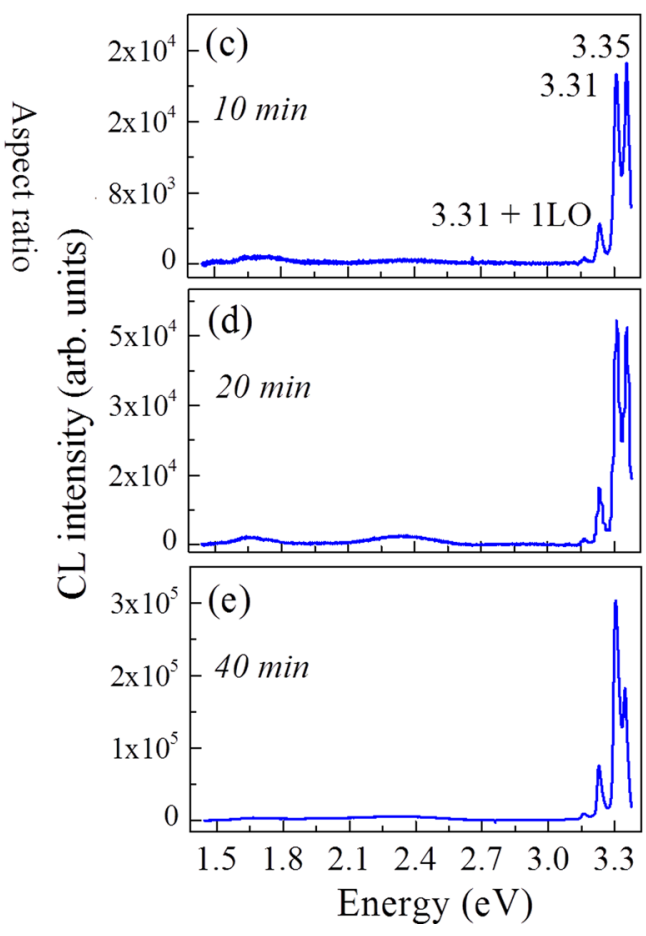

FIG. 8. (a) CL intensity of the $3.34 \mathrm{eV}$ peak and aspect ratio of $\mathrm{ZnO}$ nanorod arrays as a function of growth time; (b) Raman spectra of $\mathrm{ZnO}$ nanorod arrays grown with and without a $\mathrm{ZnO}$ buffer layer on $c$-sapphire. CL spectra of $\mathrm{ZnO}$ nanorod arrays grown with a $\mathrm{ZnO}$ buffer layer at (c) 10, (d) 20, and (e) $40 \mathrm{~min}$. 


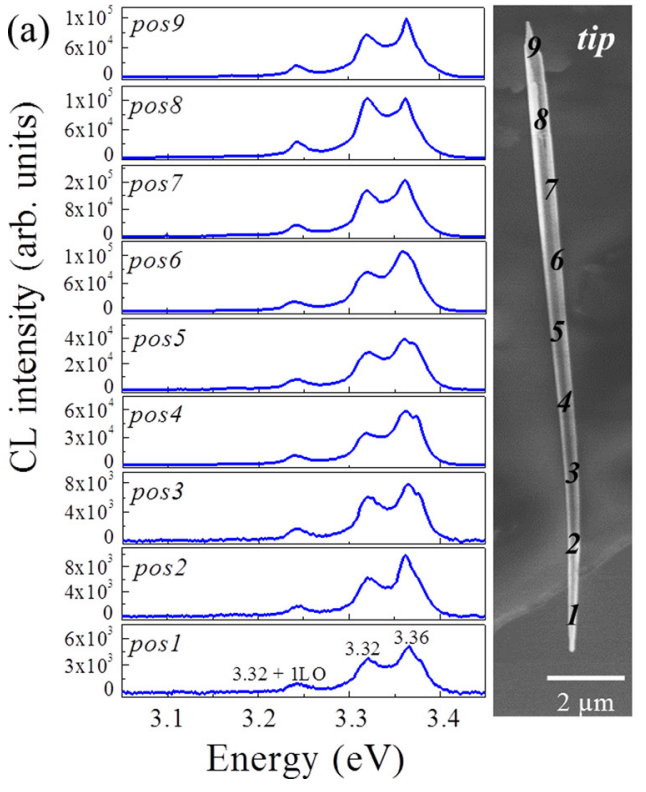

FIG. 9. CL spectra at different spatial positions along the length of individual nanorod grown on a $\mathrm{ZnO}$ buffer layer with different growth times (a) 10 and (b) $40 \mathrm{~min}$. were present in the spectrum of nanorods grown on bare sapphire substrates. This suggests that the use of a $\mathrm{ZnO}$ buffer layer tends to significantly reduce the incorporation of the defects responsible for these peaks, namely, $Z n_{I}$-related defects.

Typical CL spectra of the $\mathrm{ZnO}$ nanorods grown on buffered sapphire during 10, 20, and $40 \mathrm{~min}$ are shown in Figure 8(c). Similarly to the CL spectra of nanorods grown on bare sapphire, they exhibit the NBE emission peaks around 3.34 and $3.31 \mathrm{eV}$ with the respective phonon replicas. It is worth to remark that the $3.31 \mathrm{eV}$ band and its phonon replicas present a high intensity, which suggests a lower concentration of defects. $^{49}$

Furthermore, the intensity of the DLE luminescence is much lower than the NBE luminescence, showing a very significant reduction in comparison with the DLE luminescence from $\mathrm{ZnO}$ nanorods grown on bare $c$-sapphire. In addition, this band denotes a shift towards high energies, from red to the yellow spectral range $(\sim 2.24 \mathrm{eV})$. A shift of the DLE bands can be associated with a change in the nature of the defects responsible for the DLE luminescence. The very low red emission in nanorods grown on buffered sapphire corroborates the substantial reduction in the incorporation of a $\mathrm{Zn}$ excess, responsible for the formation of $Z n_{I}$-related defects. Yellow-orange luminescence has been associated with $V_{Z n} ;{ }^{40}$ however, the low intensity of this band in the spectra from nanorods grown on buffered sapphire and the high NBE emission suggest a low incorporation of defects in these nanorods.

Figure 9 shows SEM images and the CL spectra obtained along two individual nanorods grown on buffered sapphire during 10 and $40 \mathrm{~min}$, Figures 9(a) and 9(b), respectively. Homogeneous intensity of the NBE emission is observed along the length of both nanorods. This behavior suggests a significant suppression of NRRCs. These figures show that the luminescence is not quenched at the nanorod tip in comparison with the large volume of quenched luminescence observed in samples grown on sapphire substrates.
Both Raman and CL spectra point to a substantial reduction in the incorporation of $Z n_{I}$-defect complexes; improving thus the luminescence properties of $\mathrm{ZnO}$ nanorods, and the crystal quality, in terms of defect concentration, as observed from the intense phonon replicas all along the nanorods.

From the SEM image of Figure 9(b), it is possible to observe that the morphology of nanorods grown on a $\mathrm{ZnO}$ buffer layer is clearly different from that observed in nanorods directly grown on bare sapphire substrates. It is known that the shape of the $\mathrm{ZnO}$ nanostructures strongly depends on the structural characteristics of the $\mathrm{ZnO}$ nucleation/interfacial layer on which they grow. Therefore, the use of a $\mathrm{ZnO}$ buffer layer instead of a bare sapphire substrate during the synthesis process could substantially modify the growth mode of $\mathrm{ZnO}$ nanorods and consequently reduce the formation of $\mathrm{Zn}_{I}$-related defects.

\section{SUMMARY AND CONCLUSIONS}

A combined characterization of micro-Raman and CL spectroscopy has been used to investigate the influence of precursors partial-pressures ratio $\left(R_{V I / I I}\right)$ and growth time on the optical properties of $\mathrm{ZnO}$ nanorods grown by atmospheric pressure MOCVD. The analysis of the optical measurements made on a large series of $\mathrm{ZnO}$ nanorod arrays, grown under different oxygen- and zinc-rich conditions, has allowed to conclude that the NRRCs, which substantially affect the light emission in the visible spectral range of our nanorods, can be ascribed to $Z n_{I}$-related defects. Indeed, it has been shown that $\mathrm{Zn}$ supersaturation reduces the luminescence efficiency. The characterization of individual nanorods, grown on bare sapphire substrates, has allowed to focus on the spatial location of these defects that seem to be principally located at the nanorod tips. The measurement of individual nanorods permits to detect inhomogeneities, showing that the excess $Z n_{I}$ defects accumulate in a region extending a few micrometers below the tip. The local CL and Raman spectra reveal the incorporation of defects in that region. 
The use of a $\mathrm{ZnO}$ buffer layer in the growth process has allowed to change the morphological, luminescent, and vibrational characteristics of the $\mathrm{ZnO}$ nanorods. There is a substantial improvement of the optical quality of nanorods. Thus, the use of the $\mathrm{ZnO}$ buffer layer has allowed to substantially reduce, even for large growth times, the concentration of NRRCs, tentatively associated with $Z n_{I}$-related defects.

\section{ACKNOWLEDGMENTS}

The authors gratefully acknowledge Spanish Government for financial support under the Projects MAT2007-66129, MAT-2010-20441-C02, MAT-2010-16116, and TEC201128076-C02-02, and Generalitat Valenciana under the projects Prometeo/2011-035 and ISIC/2012/008, Institute of Nanotechnologies for Clean Energies of the Generalitat Valenciana. The authors, D. N. Montenegro, V. Hortelano, and V. Sallet thank specially to Spanish MICINN, the European Social Fund (ESF) and Universitat de Valencia, respectively, for the financial support.

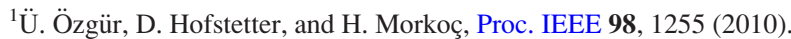

${ }^{2}$ S. Barth, F. Hernandez-Ramirez, J. D. Holmes, and A. RomanoRodriguez, Prog. Mater. Sci. 55, 563 (2010).

${ }^{3}$ M. Willander et al., Nanotechnology 20, 332001 (2009).

${ }^{4}$ A. B. Djurišić, A. M. C. Ng, and X. Y. Chen, Prog. Quantum Electron. 34, 191 (2010).

${ }^{5}$ M. Willander, O. Nur, J. Rana Sadaf, M. Israr Qadir, S. Zaman, A. Zainelabdin, N. Bano, and I. Hussain, Materials 3, 2643 (2010).

${ }^{6}$ A. B. Djurišić and Y. H. Leung, Small 2, 944 (2006).

${ }^{7}$ S. F. Chichibu, T. Onuma, M. Kubota, A. Uedono, T. Sota, A. Tsukazaki, A. Ohtomo, and M. Kawasaki, J. Appl. Phys. 99, 093505 (2006).

${ }^{8}$ H. A. Ahn, Y. Y. Kim, D. C. Kim, S. K. Mohanta, and H. K. Cho, J. Appl. Phys. 105, 013502 (2009).

${ }^{9}$ K. M. Wong, Y. Fang, A. Devaux, L. Wen, J. Huang, L. De Cola, and Y. Lei, Nanoscale 3, 4830 (2011).

${ }^{10}$ A. B. Djurišić et al., Nanotechnology 18, 095702 (2007).

${ }^{11}$ A. Janotti and C. G. Van de Walle, J. Cryst. Growth 287, 58 (2006).

${ }^{12}$ J. Conradt et al., J. Phys. Chem. C 115, 3539 (2011).

${ }^{13}$ A. C. Mofor, A. S. Bakin, A. Elshaer, D. Fuhrmann, F. Bertram, A. Hangleiter, J. Christen, and A. Waag, Appl. Phys. A 88, 17 (2007).

${ }^{14}$ B. Ling, Y. Wang, X. W. Sun, Z. L. Dong, and N. X. Yang, IEEE J. Sel. Top. Quantum Electron. 17, 801 (2011).

${ }^{15}$ F. Xu, Y. Lu, Y. Xie, and Y. Liu, J. Solid State Electrochem. 14, 63 (2010).

${ }^{16}$ C. Li, Z. Du, H. Yu, and T. Wang, Thin Solid Films 517, 5931 (2009).

${ }^{17}$ W.-C. Liu and W. Cai, Appl. Surf. Sci. 254, 3162 (2008).

${ }^{18}$ W. I. Park, Met. Mater. Int. 14, 659 (2008).

${ }^{19}$ D. J. Park, D. C. Kim, J. Y. Lee, and H. K. Cho, Nanotechnology 17, 5238 (2006).
${ }^{20}$ D. N. Montenegro, A. Souissi, M. C. Martínez-Tomás, V. Muñoz-Sanjosé, and V. Sallet, J. Cryst. Growth 359, 122 (2012).

${ }^{21}$ C. A. Arguello, D. L. Rosseau, and S. P. S. Porto, Phys. Rev. 181, 1351 (1969).

${ }^{22}$ J. M. Calleja and M. Cardona, Phys. Rev. B 16, 3753 (1977).

${ }^{23}$ R. Cuscó, E. Alarcón-Lladó, J. Ibáñez, L. Artús, J. Jiménez, B. Wang, and M. J. Callahan, Phys. Rev. B 75, 165202 (2007).

${ }^{24}$ F. Friedrich and N. H. Nickel, Appl. Phys. Lett. 91, 111903 (2007).

${ }^{25}$ A. Kaschner, U. Haboeck, M. Strassburg, M. Strassburg, G. Kaczmarczyk, A. Hoffmann, and C. Thomsen, Appl. Phys. Lett. 80, 1909 (2002).

${ }^{26}$ L. L. Kerr, X. Li, M. Canepa, and A. Sommer, Thin Solid Films 515, 5282 (2007).

${ }^{27}$ A. Marzouki et al., Mater. Lett. 64, 2112 (2010).

${ }^{28}$ C. Bundesmann, N. Ashkenov, M. Schubert, D. Spemann, T. Butz, E. M. Kaidashev, M. Lorenz, and M. Grundmann, Appl. Phys. Lett. 83, 1974 (2003).

${ }^{29}$ F. J. Manjóna, B. Marí, J. Serrano, and A. H. Romero, J. Appl. Phys. 97, 053516 (2005).

${ }^{30}$ F. Friedrich, M. A. Gluba, and N. H. Nickel, Appl. Phys. Lett. 95, 141903 (2009).

${ }^{31}$ N. H. Nickel and M. A. Gluba, J. Electron. Mater. 40, 440 (2011).

${ }^{32}$ B. K. Meyer, J. Sann, D. M. Hofmann, C. Neumann, and A. Zeuner, Semicond. Sci. Technol. 20, S62 (2005).

${ }^{33}$ D. W. Hamby, D. A. Lucca, and M. J. Klopfstein, J. Appl. Phys. 97, 043504 (2005).

${ }^{34}$ Y. Z. Zhang, H. P. He, Y. Z. Jin, B. H. Zhao, Z. Z. Ye, and H. P. Tang, J. Appl. Phys. 104, 103529 (2008).

${ }^{35}$ T. B. Hur, G. S. Jeen, Y. H. Hwang, and H. Y. Kim, J. Appl. Phys. 94, 5787 (2003).

${ }^{36}$ A. Dadgar, A. Krtschil, F. Bertram, S. Giemsch, T. Hempel, P. Veit, A. Diez, N. Oleynik, R. Clos, J. Christen, and A. Krost, Superlattices Microstruct. 38, 245 (2005)

${ }^{37}$ M. Schirra, R. Shneider, A. Reiser, G. M. Prinz, M. Biskupek, U. Kaiser, C. E. Krill, K. Thonke, and R. Sauer, Phys. Rev. B 77, 125215 (2008).

${ }^{38}$ M. A. Reshchikov, H. Morkoç, B. Nemeth, J. Nause, J. Xie, B. Hertog, and A. Osinsky, Physica B 401-402, 358 (2007).

${ }^{39}$ K. Vanheusden, W. L. Warren, C. H. Seager, D. R. Tallant, J. A. Voigt, and B. E. Gnade, J. Appl. Phys. 79, 7983 (1996).

${ }^{40}$ M. Liu, A. H. Kitai, and P. Mascher, J. Lumin. 54, 35 (1992).

${ }^{41}$ T. Monteiro, C. Boemare, M. J. Soares, E. Rita, and E. Alves, J. Appl. Phys. 93, 8995 (2003).

${ }^{42}$ Y. F. Mei, G. G. Siu, K. Y. Fu Ricky, K. W. Wong, K. Chu Paul, C. W. Lai, and H. C. Ong, Nucl. Instrum. Methods Phys. Res. B 237, 307 (2005).

${ }^{43}$ M. C. Tarum, M. Zafar Iqbal, and M. D. McCluskey, AIP Adv. 1, 022105 (2011).

${ }^{44}$ M. Gomi, N. Oohira, K. Ozaki, and M. Koyano, Jpn. J. Appl. Phys., Part 1 42, 481 (2003).

${ }^{45}$ K. E. Knutsen, A. Galeckas, A. Zubiaga, F. Tuomisto, G. C. Farlow, B. G. Svensson, and A. Y. Kuznetsov, Phys. Rev. B 86, 121203R (2012).

${ }^{46}$ V. Sallet, S. Agouram, F. Falyouni, A. Marzouki, N. Haneche, C. Sartel, A. Lusson, S. Enouz-Vedrenne, V. Munoz-Sanjose, and P. Galtier, Phys. Status Solidi B 247, 1683 (2010).

${ }^{47}$ M. C. Martínez-Tomás, D. N. Montenegro, V. Sallet, and V. MuñozSanjosé, J. Appl. Phys. 112, 014305 (2012).

${ }^{48}$ D. C. Kim, B. H. Kong, H. K. Cho, D. J. Park, and J. Y. Lee, Nanotechnology 18, 015603 (2007).

${ }^{49}$ J. Mass, M. Avella, J. Jimenez, A. Rodriguez, T. Rodriguez, M. Callahan, D. Bliss, and B. Wang, J. Cryst. Growth 310, 1000 (2008) 